Classification

Physics Abstracts

$72.15 \mathrm{G}$

\title{
Shubnikov-de Haas oscillations in an organic conductor tetramethyltetraselenafulvalene-2,5-dimethyl-7,7', 8,8' tetracyanoquinodimethane (TMTSF-DMTCNQ)
}

\author{
S. Bouffard, \\ Section d'Etude des Solides Irradiés, CEN, B.P. 6, 92260 Fontenay-aux-Roses, France \\ M. Ribault, D. Jérome and K. Bechgaard \\ Laboratoire de Physique des Solides, Université de Paris-Sud, 91405 Orsay, France
}

(Reçu le 3 décembre 1982, accepté le 28 février 1983)

\begin{abstract}
Résumé. - L'observation d'oscillations de Haas-Shubnikov dans la phase métallique (Metal-II) de TMTSF-DMTCNQ stabilisée sous haute pression $(P>10 \mathrm{kbar})$ démontre le caractère tridimensionnel de la surface de Fermi à basse température $(T<2 \mathrm{~K})$. Les sections de la surface de Fermi sont respectivement de $1,16 \times 10^{17}$ et $2,6 \times 10^{17} \mathrm{~m}^{-2}$ pour les plans $a b$ et $a c$. La masse cyclotron $m_{\mathrm{c}} / m_{0} \simeq 0,56$ et la température de Dingle $T_{\mathrm{D}}=0,8 \mathrm{~K}$ sont obtenues à partir de la dépendance des oscillations $\mathrm{SdH}$ en fonction du champ et de la température. Enfin, nous examinons ces résultats en fonction de la conductivité électronique qui s'élève à $3 \times 10^{5}(\Omega \mathrm{cm})^{-1}$ à $T=1,2 \mathrm{~K}$.
\end{abstract}

\begin{abstract}
The observation of Shubnikov-de Haas (SdH) oscillations in the metallic, high pressure phase (Metal-II) of TMTSF-DMTCNQ $(P>10 \mathrm{kbar})$ demonstrates the 3-dimensional, closed nature of the Fermi surface at low temperature $(T<2 \mathrm{~K})$. We observe very small Fermi surface crosssections of $1.16 \times 10^{17} \mathrm{~m}^{-2}$ and $2.6 \times 10^{17} \mathrm{~m}^{-2}$ in the $a b$ and $a c$ planes respectively. From the field and temperature dependence of the $\mathrm{SdH}$ oscillations we extract a cyclotron mass $m_{\mathrm{c}} / m_{0} \simeq 0.56$ and a Dingle temperature $T_{\mathrm{D}}=0.8 \mathrm{~K}$. Finally we discuss the discrepancy between these results and the very high de conductivity $\left(\sigma_{1.2 \mathrm{~K}} \simeq 3 \times 10^{5}(\Omega \mathrm{cm})^{-1}\right)$.
\end{abstract}

The topology of the Fermi Surface (FS) of quasi one dimensional (Q-1-D) organic conductors is a topic of current interest [1]. In materials consisting of stacks of planar molecules one may a priori expect an open FS, but with some deviation from planarity, related to the interchain hopping integral $t_{\perp}$. This parameter is essential if we want to describe for example the observation of superconductivity in such materials, because it is used to account for a finite Josephson coupling $\left(t_{\perp}^{2} / t_{\|}\right)$between the chains and thus allows a long range ordered superconducting state at finite temperature.

In some cases however the FS of Q-1-D conductors do display closed orbits. Consequently 
the models used to describe the transport properties change from the 1-D picture with chains coupled by weak tunneling to a more conventional 2D (or 3D) picture.

A closed FS in Q-1-D conductors has been observed in two phenomenologically different situations.

(i) In two-chain compounds such as HMTSF-TCNQ the closed orbits is a result of hybridization of electronic states [2-4] with wave vectors near $\pm k_{\mathrm{F}}$ on the two different chains.

(ii) Closed orbits have been reported in single chain conductors (the $\mathrm{TMTSF}_{2} \mathrm{X}$-family), but in these materials they are observable only after a magnetic field induced phase transition has occurred [5].

HMTSF-TCNQ is so far the only two-chain material where Shubnikov-de Haas oscillations in the magnetoresistance have been observed [6]. In this material is was argued that short Se-N contacts are weakening the Peierls instability occurring at $24 \mathrm{~K}$ [7]. Thus a gap does not extend over the whole FS and this leads to semimetallic behaviour at helium temperature. At the same time lattice disorder and a hydrostatic pressure of 4 kbar can suppress the transition, but the maximum conductivity observable at low temperature is of the order of $10^{4}(\Omega \mathrm{cm})^{-1}[8]$.

The material TMTSF-DMTCNQ at ambient pressure undergoes a Peierls transition at $41 \mathrm{~K}$ [9]. The conducting phase, stable down to $41 \mathrm{~K}$ at ambient pressure, has been called the Metal-I phase.

A hydrostatic pressure of about $10 \mathrm{kbar}$ abruptly suppresses the Peierls insulating state [10]. The predominant feature of the socalled Metal-II state of TMTSF-DMTCNQ (at $P>10 \mathrm{kbar}$ ) is a conductivity at least one order of magnitude larger than that of HMTSF-TCNQ [8].

In the present work we have determined the FS topology of TMTSF-DMTCNQ in the Metal-II state, by observing $\mathrm{SdH}$ oscillations in magnetic fields transverse to the highly conducting (a) axis. A highlight of this work is the derivation, from the $\mathrm{SdH}$ measurements of a single particle conductivity which is several orders of magnitude smaller than the observed values of $3 \times$ $10^{5}(\Omega \mathrm{cm})^{-1}$ at helium temperature [10]. We propose an interpretation of this discrepancy in terms of a contribution of short range 1-D superconducting fluctuations.

\section{Experimental.}

The magnetoresistance of high purity TMTSF-DMTCNQ single crystals was measured by a classical four contacts and low frequency method. The resistance was recorded versus magnetic field, without field modulation. The samples were pressurized up to $12 \mathrm{kbar}$ in a copper-berylium cell and cooled slowly in a dilution refrigerator. The magnetic field (up to 7 Tesla) was aligned either along the $b$ or $c$ direction of the crystals. The accuracy of the alignment was not better than 10 degrees.

\section{Results.}

As previously reported, [10] a dramatic increase of the conductivity is observed on cooling. In this work our best result was $\sigma(1.2 \mathrm{~K}) / \sigma(300 \mathrm{~K})=135$ at $P=12 \mathrm{kbar}$. Together with the pressure increase at room temperature by a factor 4.75 between ambient and $12 \mathrm{kbar}$ pressure, the conductivity at low temperature culminates at $\simeq 3 \times 10^{5}(\Omega \mathrm{cm})^{-1},[\sigma(300 \mathrm{~K}, 1$ bar $) \approx$ $\left.500(\Omega \mathrm{cm})^{-1}\right]$.

In spite of the large and still somewhat temperature dependent resistivity at helium temperature the present study and some previous attempts have failed to detect superconductivity down to $80 \mathrm{mK}$.

Raw magnetoresistance data are displayed on figure 1 , for temperatures from $155 \mathrm{mK}$ to $3 \mathrm{~K}$ and with the magnetic field along the $c$-axis. The oscillations are most clearly observed below $1 \mathrm{~K}$, but their amplitude never grows above a few percent of the non-oscillating background. Data qualitatively similar were obtained with the magnetic field along the $b$-direction. 


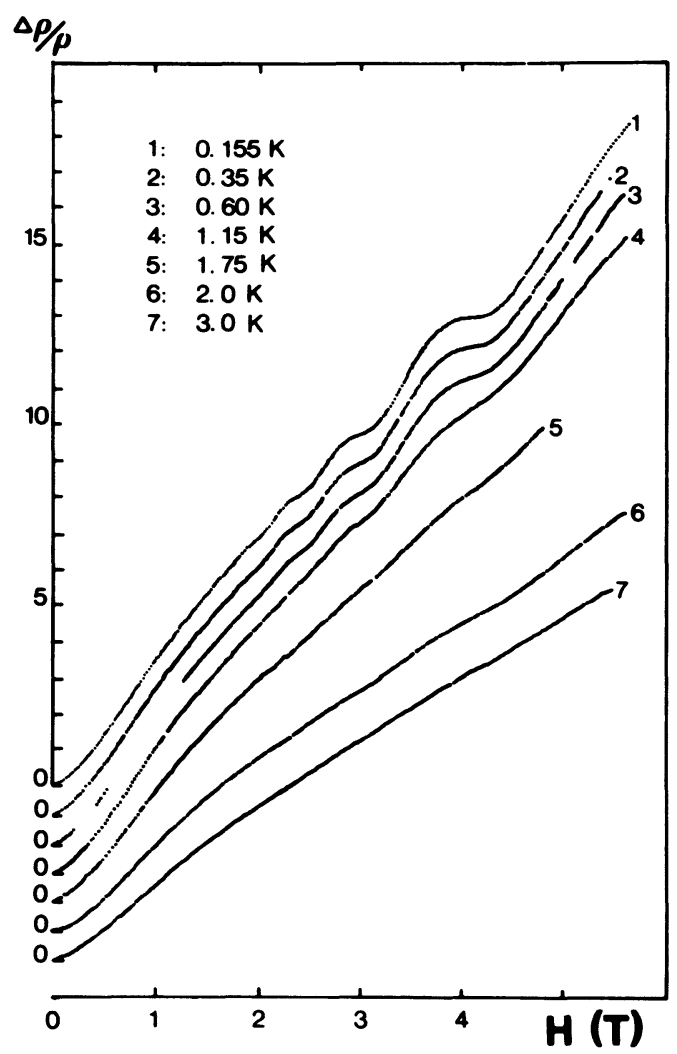

Fig. 1. - Magnetoresistivity of TMTSF-DMTCNQ under $12 \mathrm{kbar}$ at different temperatures. The applied magnetic field is parallel to the $c$-axis.

The results of figure 1 clearly indicate a quantum origin to the high-field magnetoresistance. Indeed they can be analysed following the expression of the SdH effect [11] :

$$
\frac{\Delta \rho}{\rho} \approx\left(\frac{\hbar S_{\mathrm{m}}}{2 \pi e B}\right)^{-1 / 2} \cdot \sum_{r=1}^{\infty} \frac{(-1)^{r}}{r^{1 / 2}} \cdot \frac{k T m_{\mathrm{c}}}{\hbar e B} \cdot \frac{\exp \left(-\frac{2 \pi^{2} r m_{\mathrm{c}} k T_{\mathrm{D}}}{\hbar e B}\right)}{\sinh \left(\frac{2 \pi^{2} r m_{\mathrm{c}} k T}{\hbar e B}\right)} \cdot \cos \left(\frac{r \hbar S_{\mathrm{m}}}{e B}-\frac{\pi}{4}\right)
$$

where $m_{\mathrm{c}}$ is the cyclotron mass, $S_{\mathrm{m}}$ the extremal cross section of the FS in a plane perpendicular to the magnetic field and $T_{\mathrm{D}}$ the Dingle temperature $\left(k T_{\mathrm{D}} \sim \hbar / \pi \tau\right)$.

Magnetic fields corresponding to minima and maxima of the oscillations are plotted versus integers and half-integers in figure 2. The plot shows that equation 1 accounts well for the oscillatory nature of the magnetoresistance. Furthermore, the results of figure 2 allows a determination of the periods $0.086 \mathrm{~T}^{-1}$ and $0.24 \mathrm{~T}^{-1}$ for the oscillations with $B$ along the $c$ and $b$-axis respectively. The corresponding cross-sections $1.16 \times 10^{17} \mathrm{~m}^{-2}$ and $2.6 \times 10^{17} \mathrm{~m}^{-2}$ in the $(a-b)$ and (a-c) planes respectively amount to about $0.1 \%$ of a Brillouin zone cross section.

The measurement of the temperature dependence of the amplitude at fixed field, figure 3, gives an estimate of the cyclotron mass whereas the field dependence at constant temperature leads to $T_{\mathrm{D}}$. We obtain $m_{\mathrm{c}}=(0.56 \pm 0.05) m_{0}$ and $T_{\mathrm{D}} \sim 0.8 \mathrm{~K}$ for $B / / c$. 


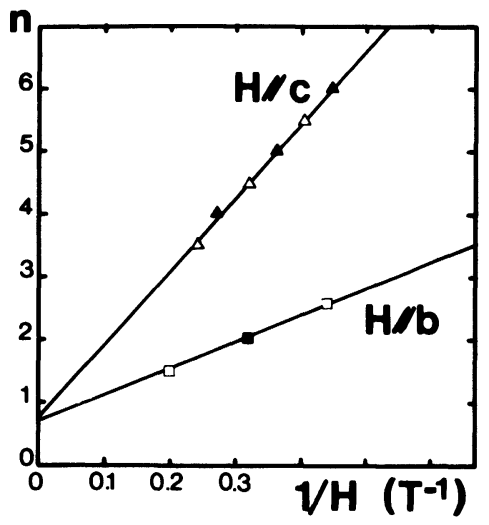

Fig. 2. - Successive integers and half-integers corresponding to maxima and minima of the oscillations versus $H^{-1}$. The upper curve is for $H / / c$ and the lower for $H / / b$.

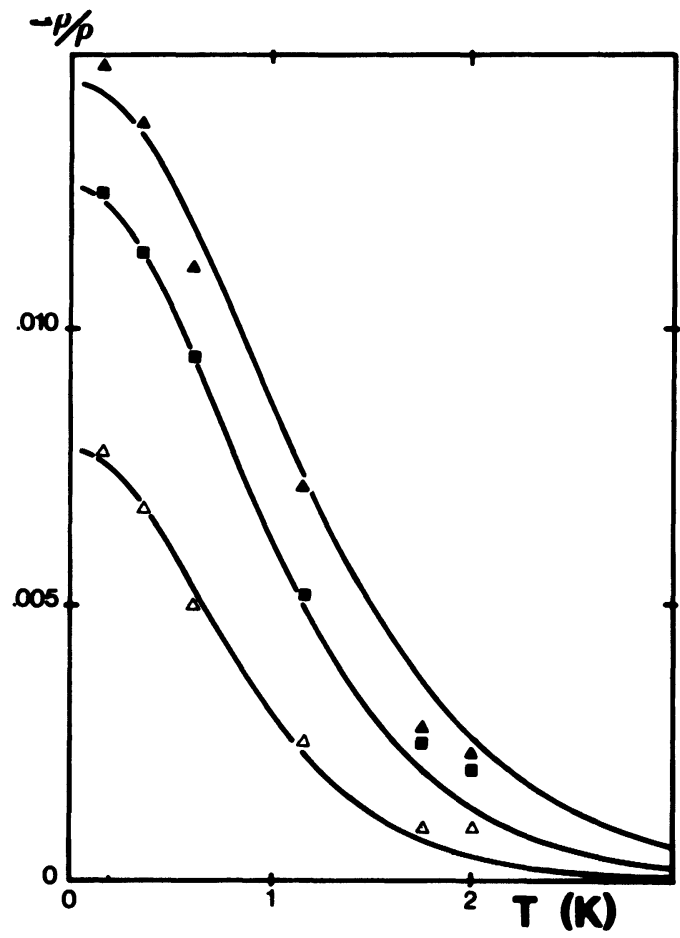

Fig. 3. - Amplitude of three extrema (4.42, 3.7 and 3.14 Tesla) of the oscillations as a function of the temperature. The solid lines show the theoretical results with a cyclotron mass of $0.56 \mathrm{~m}_{0}$.

As demonstrated in figure 4 the field and temperature dependence of the oscillations is in agreement with the derived values of $m_{c}$ and $T_{\mathrm{D}}$.

In table I, we compare the parameters of TMTSF-DMTCNQ with the parameters obtained for HMTSF-TCNQ [6] and (TMTSF) ${ }_{2} \mathrm{PF}_{6}$ [5]. We emphasize that SdH oscillations of the 1-chain materials are observed only above a threshold field, the amplitude of which depends on the orientation with respect to crystal axis and temperature. In (TMTSF) ${ }_{2} \mathrm{PF}_{6}$ for instance, the 


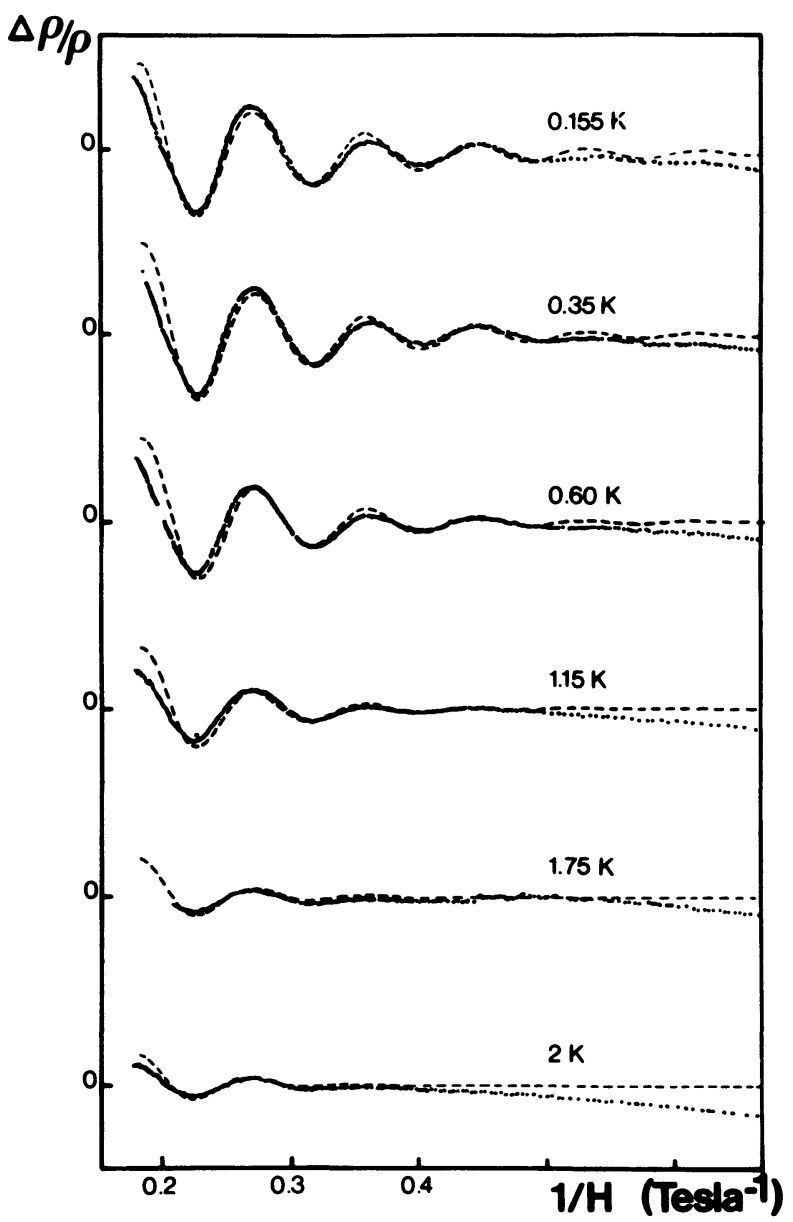

Fig. 4. - Oscillatory magnetoresistivity of TMTSF-DMTCNQ under $12 \mathrm{kbar}(H / / c)$ versus $1 / \mathrm{H}$ for different temperatures. The solid lines show the best fit (see the text).

threshold field is $65 \mathrm{kOe}\left(\mathrm{H} / / \mathrm{c}\left({ }^{1}\right)\right)$ at $1 \mathrm{~K}$ under $7 \mathrm{kbar}$ [5]. A similar threshold field effect has been reported for (TMTSF) ${ }_{2} \mathrm{ClO}_{4}$ under a very small pressure [12] or after slow cooling at ambient pressure. No threshold field is observed for TMTSF-DMTCNQ and HMTSF-TCNQ.

Table I.

\begin{tabular}{|l|c|c|c|}
\cline { 2 - 4 } \multicolumn{1}{c|}{} & $S_{\mathrm{m}}\left(10^{16} \mathrm{~m}^{-2}\right)$ & $m_{\mathrm{c}} / m_{0}$ & $T_{\mathrm{D}}(\mathrm{K})$ \\
\hline TMTSF-DMTCNQ & $\begin{array}{l}11.6(/ / c) \\
26.7(/ / \mathrm{b})\end{array}$ & 0.56 & 0.8 \\
\hline HMTSF-TCNQ & $7(/ / c)$ & 0.05 & $(1)\left({ }^{1}\right)$ \\
\hline$(\mathrm{TMTSF})_{2} \mathrm{PF}_{6}$ & $73(/ / c)$ & 1.1 & 3 \\
\hline
\end{tabular}

( ${ }^{1}$ ) The Dingle temperature was not reported in reference [6] but the domain of temperatures in which oscillations are observed is consistent with $T_{\mathrm{D}} \approx 1 \mathrm{~K}$. 


\section{Interpretation.}

The data of the present work prove conclusively the existence of closed orbits on the FS of the Metal-II phase of TMTSF-DMTCNQ at low temperature. These closed orbits are provided by nearly ellipsoidal pockets of very small volume. If we assume parabolic bands and use the SdH parameters (Table I) and figure 2 we obtain the Fermi energy of a pocket $\varepsilon_{\mathrm{F}} \approx 50 \mathrm{~K}$. Moreover, the volume enclosed within such small pockets is about $3 \times 10^{-4}$ times smaller than the volume enclosed by the original high temperature 1-D Fermi surface. Thus, the density of carriers seen by $\mathrm{SdH}$ effects at low temperature in the Metal-II phase is about $10^{-4}$ per formula-unit, i.e. $3 \times 10^{18} \mathrm{~cm}^{-3}$ (assuming two pockets of such carriers per Brillouin zone). The Fermi energy which can be derived from the density of carriers and the data in table I, $\varepsilon_{F}=\frac{\hbar^{2}}{2 m^{*}}\left(3 \pi^{2} n\right)^{2 / 3}$, within a nearly free electron approximation $\left(m^{*} \sim 0.5 m_{0}\right)$, amounts to $\approx 180 \mathrm{~K}$.

We infer that the present $\mathrm{SdH}$ results can be interpreted within the framework of the model [4] proposed for the interpretation of the galvanomagnetic data of HMTSF-TCNQ at low temperature [13]. In this model a 1-D to 3-D band structure transformation occurring gradually upon cooling to low temperature was suggested.

Starting our description with a (hypothetical) situation where we assume a zero transverse coupling between adjacent 1-D chains, the bands derived from the donor and acceptor chains respectively, will cross at the Fermi level and the FS is planar. This planar geometry is however slightly modified by the conjugated effects of weak couplings both between like and unlike chains (Q-1-D situation) which transforms the original planar FS derived from non-interacting chains into a semimetallic three-dimensional FS. The semimetallic FS appears as small pockets of electrons and holes comprising a volume which is much smaller than the original Brillouin zone. The energy spread of the small pockets is typically of the order of $t_{\perp}$. Therefore as long as the smearing $\hbar / \tau_{\|}$of the FS by the finite electron life time $\tau_{\|}$is larger than $t_{\perp}$, we do not expect to observe 3-D Fermi surface characteristics. Consequently, a semimetallic FS may be observed only when the condition

$$
\hbar \tau_{\|}^{-1}<t_{\perp}
$$

is fulfilled.

The condition $k T<t_{\perp}$ must also be fulfilled, but we expect this latter condition to be less restrictive since at low temperature the growth of $\tau_{\|}$is limited by residual impurities and $k T$ becomes smaller than $\hbar / \tau_{\|}$. The finding of $t_{\perp} \approx \varepsilon_{\mathrm{F}} \approx 50 \mathrm{~K}$ from the SdH data shows a posteriori that the starting assumption of a Q-1-D Metal-II phase is verified, because unless $t_{\|}$is " accidentally 》 diminished significantly under high-pressure, we obtain $t_{\perp} \ll t_{\|}$.

Other experimental data corroborate the existence of small pockets in the FS of TMTSFDMTCNQ under pressure and at low temperature.

First, the thermopower at $12 \mathrm{kbar}$ [10] exhibits semimetallic behaviour below $10 \mathrm{~K}$ showing in addition that the dominant carriers are holes.

$\varepsilon_{\mathrm{F}}$ is related to the Seebeck coefficient $S$ by $S=\frac{\pi^{2}}{2} \frac{k}{e} \frac{k T}{\varepsilon_{\mathrm{F}}}$ and $S=\frac{\pi^{2}}{6} \frac{k}{e} \frac{k T}{\varepsilon_{\mathrm{F}}}$ in 3-D and 1-D band models respectively [14], with corresponding $\varepsilon_{\mathrm{F}}(3 \mathrm{D})=300 \mathrm{~K}$ and $\varepsilon_{\mathrm{F}}(1 \mathrm{D})=100 \mathrm{~K}$. Both values are larger than the estimate $50 \mathrm{~K}$ based on the $\mathrm{SdH}$ oscillations. This apparent discrepancy is discussed below. Secondly the magnetic susceptibility of TMTSF-DMTCNQ shows strong diamagnetism under pressure at low temperature (below $30 \mathrm{~K}$ ) with $\chi_{\text {dia }}$ reaching $-7 \times$ $10^{-4} \mathrm{emu} / \mathrm{mole}$ at $4.2 \mathrm{~K}[15]$. This behaviour was interpreted as the signature of a small FS giving rise to a strong Landau-Peierls diamagnetism.

The question which arises now is : how can the very few carriers of TMTSF-DMTCNQ explain 
the highest conductivity ever found at helium temperature in an organic conductor (besides organic superconductors)?

The Dingle temperature of $0.8 \mathrm{~K}$ gives a fairly long mean scattering time $\tau \sim 3 \times 10^{-12} \mathrm{~s}$, about 100 times longer than the optical scattering times obtained from reflectance studies with light polarized along the chain axis. These measurements have not been done for TMTSFDMTCNQ under pressure, but results are available for compounds exhibiting a similar high dc conductivity at low temperature. For instance $\tau=10^{-14} \mathrm{~s}$ and $1.5 \times 10^{-14} \mathrm{~s}$ in (TMTSF) ${ }_{2} \mathrm{PF}_{6}$ at $25 \mathrm{~K}[16]$ and (TMTSF) ${ }_{2} \mathrm{ClO}_{4}$ at $30 \mathrm{~K}$ [17] respectively.

Since the optical reflectance and the SdH effect refer to different shapes of the FS, the factor 100 is not surprising. The semimetallic carriers have long scattering time (but a much reduced Fermi velocity) and the mean free-path $\Lambda=v_{\mathrm{F}} \tau$ does not vary much through the 1-D to 3-D FS crossover. Following the definition $\sigma=n e^{2} \tau / m^{*}$ the semimetallic conductivity amounts to about $1500(\Omega \mathrm{cm})^{-1}, m^{*}=0.5 m_{0}, n=3 \times 10^{18} \mathrm{~cm}^{-3}, \tau=3 \times 10^{-12} \mathrm{~s}$. This value may be underestimated by one order of magnitude or so because $\tau$ derived from SdH oscillations is frequently shorter than $\tau$ derived from dc conductivity. We are however still far from the experimental conductivity of $3 \times 10^{5}(\Omega \mathrm{cm})^{-1}$.

We may consider other carriers, not revealed by oscillatory magnetoresistance, as the source of the high conductivity. However we find this possibility rather unlikely since with the model of band hybridization [4] the density of carriers (electrons) must be similar to the density of holes, i.e. $\approx 3 \times 10^{18} \mathrm{~cm}^{-3}$. Furthermore the former carriers which do not show up in $\mathrm{SdH}$ oscillations have presumably a mass larger than $0.5 \mathrm{~m}_{0}$. Therefore, unless their relaxation time is accidentaly very long $\tau \sim 10^{-9} \mathrm{~s}$, they cannot explain the conductivity of $3 \times 10^{5}(\Omega \mathrm{cm})^{-1}$.

Consequently, the semimetallic picture which can explain the low temperature state of HMTSFTCNQ $\left(\sigma \sim 10^{3}-10^{4}(\Omega \mathrm{cm})^{-1}\right)$ fails to give a reasonable estimate of the extraordinary large value of the conductivity of TMTSF-DMTCNQ.

In order to explain the intriguing question of extra conduction in TMTSF-DMTCNQ a contribution provided from short range 1-D superconducting pairing has been suggested [10]. This fluctuating channel $\sigma^{\prime}$ acts in parallel with the single particle channel $\sigma_{0}$ and the total conductivity becomes

$$
\sigma=\sigma^{\prime}+\sigma_{0}
$$

The strong sensitivity of the $\sigma^{\prime}$ channel to a low concentration of irradiation induced defects has allowed a qualitative evaluation of the contributions [19]. At helium temperature $\sigma^{\prime} / \sigma_{0} \sim 10-20$ and $\sigma^{\prime}$ is dominant in the definition (3) below $30 \mathrm{~K}$.

Therefore, as far as $\mathrm{SdH}$ experiments are concerned the paraconductivity $\sigma^{\prime}$ could account for the high dc conductivity and its large field dependence, whereas the small hole pockets would provide the small amplitude magnetoresistance oscillations. When the concept of superconducting fluctuations is introduced into the definition of the thermopower a two-fluids model can be used. The thermopower thus reads :

$$
S=\frac{\sigma^{\prime} S^{\prime}+\sigma_{0} S_{0}}{\sigma^{\prime}+\sigma_{0}}
$$

On the one hand the fluctuating part of the thermopower $S^{\prime}$ is expected to be small if not zero, since no heat is carried by a current of superconducting origin. On the other hand the normal contribution, which follows a linear $T$-dependence can reach fairly large values in a semimetal with a very small Fermi energy. Consequently equation (4) reduces to :

$$
S=\frac{\sigma_{0}}{\sigma^{\prime}} S_{0},
$$


and the Fermi energy derived from the thermopower data drops to $\varepsilon_{\mathrm{F}} \approx 30 \mathrm{~K}$ (with the 3-D approximation) in much better agreement with the $\mathrm{SdH}$ results.

The final question, to which this article is adressed, is why no superconductivity is observed in TMTSF-DMTCNQ ?

There are no clear answers to this question, but we may suggest some possibilities :

(i) The phase of TMTSF-DMTCNQ which shows at low temperature the high conductivity behaviour is the Metal-II phase, of which very little is known. This phase may be incommensurate since pressure is known to enhance the band filling in two-chain conductor [20,21]. It is characteristic that the (TMTSF) ${ }_{2} \mathrm{X}$ series in which superconductivity is observed, is commensurate. Commensurability seems to be relevant for the stabilization of superconductivity through electron Umklapp scattering $[22,23]$, thus possible effects of non-commensurability should be considered for the phase Metal-II of TMTSF-DMTCNQ.

(ii) Weak and non-magnetic defects suppress very easily the 3-D ordered superconducting state of Q-1-D superconductors with much less perturbation of the 1-D divergence [24, 25]. Therefore, the possible existence of such defects in the phase II should also be considered.

(iii) In the semimetallic state the density of carriers is very small and the Coulomb interaction is too weakly screened to allow the establishment of superconductivity.

\section{Conclusion.}

In summary, the present report of well-defined $\mathrm{SdH}$ oscillations in an organic conductor have bearing on several key questions regarding its electronic properties. The coupling between molecular orbitals of like and unlike chains transforms the high temperature planar FS into a low temperature semimetallic 3-D surface. Direct observation of the FS surface at low temperature is possible because a highly conducting state is stabilized under pressure. The reason for the suppression of the Peierls state of TMTSF-DMTCNQ under pressure is still an unresolved problem. Horovitz et al. [26] have suggested that an enhancement of the transverse coupling under pressure can lead to a suppression of the Peierls transition in a Q-1-D conductor when $t_{\perp}$ becomes comparable with $t_{\|}$say $t_{\perp} \approx(0.2-0.5) t_{\|}$.

We consider this model inapplicable to account for the suppression of the Peierls state of TMTSF-DMTCNQ since the present SdH studies show that the band structure of the Metal-II phase appears to be nearly one-dimensional $\left(t_{\perp} / t_{\|} \ll 1\right)$.

With these results in mind we reemphasize that the semimetallic two-dimensional Fermi surface of the (TMTSF $)_{2} \mathrm{X}$ series is related to the occurrence of magnetic ordering driven by a magnetic field above $50 \mathrm{kOe}(\mathrm{H} / / \mathrm{c})$ [27].

We also emphasize that in the one chain materials such as (TMTSF) ${ }_{2} \mathrm{X}$ a closed FS would require a much stronger $t_{\perp}$. In the present two chains material, the closed FS is a result of interband hybridization; even though $t_{\perp}$ is very small.

Finally the low density of carriers in TMTSF-DMTCNQ fails to explain the experimental conductivity of $3 \times 10^{5}(\Omega \mathrm{cm})^{-1}$ at low temperature $(P=12 \mathrm{kbar})$. The coexistence of a fluctuating superconducting contribution, important below $30 \mathrm{~K}$ [28], with a single-particle contribution is not contradictory with the present results.

\section{Acknowledgments.}

We thank L. Forro for useful discussion and D. Mailly for his help in the experiment. 


\section{References}

[1] For a recent review of the electronic properties of organic conductors see : JÉrome, D. and SCHULz, H. J., Adv. Phys. 31 (1982) 299, and Friedel, J. and Jérome, D., Contemp. Phys. 23 (1982) 583.

[2] Bernstein, U., Chaikin, P. M. and Pincus, P., Phys. Rev. Lett. 34 (1975) 271.

[3] Berlinsky, A. J., Cardan, J. F. and Weiler, L., Solid State Commun. 15 (1974) 795.

[4] Weger, M., Solid State Commun. 19 (1976) 1149.

[5] Kwak, J. F., Schirber, J. E., Greene, R. L. and Engler, E. M., Phys. Rev. Lett. 46 (1981) 1296.

[6] Miljak, M., Andrieux, A., Friend, R. H., Malfait, G., Jérome, D. and BechgaArd, K., Solid State Commun. 26 (1978) 969.

[7] Pouget, J. P., Chemica Scripta 17 (1981) 85.

[8] CoOper, J. R., Weger, M., Jérome, D., Lefur, D., BechgaARd, K., Bloch, A. N. and Cowan, D. O., Solid State Commun. 19 (1976) 749.

[9] Jacobsen, C. S., Mortensen, K., Andersen, J. R., BechgaArd, K., Phys. Rev. B 18 (1978) 905.

[10] Andrieux, A., Chaikin, P. M., Duroure, C., Jérome, D., Weyl, C., BechgaArd, K., Andersen, J. R., J. Physique 40 (1979) 1199.

[11] Roth, L. M. and ARgYres, P. N., in Semiconductors and Semimetals, R. K. Willardson and A. C. Beer (Ac. Press) 1966 vol. 1, p. 159.

[12] KwaK, J. F., Schirber, J. E., Greene, P. L. and Engler, E. M., Mol. Cryst. Liq. Cryst. 79 (1982) 111.

[13] JÉRome, D. and Weger, M., Chemistry and Physics of One-dimensional Metals, H. J. Keller editor (Plenum Press, New York) 1977, p. 341.

[14] Chaikin, P. M., The Physics and Chemistry of Low Dimensional Solids, L. Alcacer editor (D. Reidel Publ. Comp., Dordrecht) 1980, p. 53.

[15] Hardebush, U., Gerhardt, W., Schilling, J. S., Bechgaard, K., Weger, M., Miljak, M., Cooper, J. R., Solid State Commun. 32 (1979) 1151.

[16] Jacobsen, C. S., TANner, D. B., BechgaArd, K., Phys. Rev. Lett. 46 (1981) 1142.

[17] KikUChI, K., IKernoto, I., Yabushi, K., KURoda, H., Kobayashi, K., Solid State Commun. 42 (1982) 433.

[18] Wampler, W. R. and SPringford, M., J. Phys. C 5 (1972) 2345.

[19] Bouffard, S., Ribault, M., Brusetti, R., Jérome, D. and Bechgaard, K., J. Phys. C 15 (1982) 2951.

[20] Friend, R. H., MiljaK, M. and Jérome, D., Phys. Rev. Lett. 40 (1978) 1048.

[21] Megtert, S., Comès, R., Vettier, C., Pynn, R., Garito, A. F., Mol. Cryst. Liq. Cryst. 85 (1982) 159.

[22] Horovitz, B., Gutfreund, H. and Weger, M., Solid State Commun. 39 (1981) 541.

[23] Emery, V. J., Bruinsma, R., Barisic, S., Phys. Rev. Lett. 48 (1982) 1039.

[24] Larkin, A. I. and Melnikov, V. I., Sov. Phys. JETP 44 (1976) 1159.

[25] Schulz, H. J. and Bourbonnais, C., preprint (1982).

[26] Horovitz, B., Gutfreund, H., Weger, M., Phys. Rev. B 12 (1975) 3174.

[27] Takahashi, T., Jérome, D. and BechgaArd, K., J. Physique Lett. 43 (1982) L-565.

[28] Schulz, H. J., Jérome, D., Mazaud, A., Ribault, M., BechgaArd, K., J. Physique 42 (1981) 991. 